\title{
Intelligent and communicating real-time diagnostics for the fight against the Fire Compartments.
}

\author{
B.Patte-Rouland ${ }^{1}$, B.Betting ${ }^{1}$, M.Lebey ${ }^{2}$, E.Varea $^{1}$ \\ ${ }^{1}$ Université Rouen Normandie, CORIA UMR 6614, 76801 Saint-Etienne du Rouvray \\ ${ }^{2}$ Université du Havre Normandie, LOMC CNRS UMR 6294 \& 2*LITIS EA 4108, 76600 Le havre
}

\begin{abstract}
This results aims to improve the effectiveness of security systems by optimizing the interaction between technological components and organizational and human intervention. Objectives are to determine the specific laws governing indoor fire phenomena by means of experimental full-scale and numerical approaches, to get real-time information about the fire during operations. A second phase consists in a transformation of the usual passive-safety systems into intelligent and communicating systems, thereby optimizing and securing the human and organizational emergency response. The dual competence numerical /experimental data is essential in this type of study since the experimental data suffer from a lack of resolution (spatial, temporal) but nevertheless represent information necessary for validating the codes.
\end{abstract}

\section{INTRODUCTION}

During indoor fires, the decision for emergency responses is mainly based on human observations coming only from firefighters experiences without any solution to obtain precise physical information about the evolution of the fire. In compartment firefighting, the ability to have physical measurements about the nature of the fire and its evolution, and to transmit them outside for analysis in order to point out the main characteristics of the fire and detect dangers, would make up essential elements to gain a better understanding of the situation, and consequently to allow a better organization of firerescue actions thus improving firefighters security. This is the main challenge of this project, whose objectives are to determine the specific laws governing indoor fire phenomena by means of experimental full-scale and numerical approaches, to get real-time information about the fire during operations [1-5]. A second phase consists in a transformation of the usual passive-safety systems into intelligent and communicating systems, thereby optimizing and securing the human and organizational emergency response. The project focuses on several areas of research that aim to improve diagnostic capabilities in real time and continuously in order to optimize support to operational decisions in real time in the fight against fires compartments. As presented in Section context, smoke plays a major role. So obviously they are dangerous, they still have the advantage of carrying characteristic information of the nature and dynamics of accidents, physical information that can be collected to build support for computer-aided operational decisions [6-8].
This project therefore requires knowing the following areas and to analyze the interactions :

- The fire dynamics developing in semi-confined complex configuration spaces, or fire compartments

- The movement of smoke in these types of fires, and control methods,

- The fundamental laws of combustion fireplaces fire compartments

- Methods and means of detection and monitoring of the evolution of these types of fires, with the study of the associated sensors, optimization of sensors and their location to allow a better assessment of the situation and its evolution in space and in time,

- Methods and means of transmission Optical data transmission to a control center.

\section{Experimental and numerical set-up}

\subsection{Experimental cell}

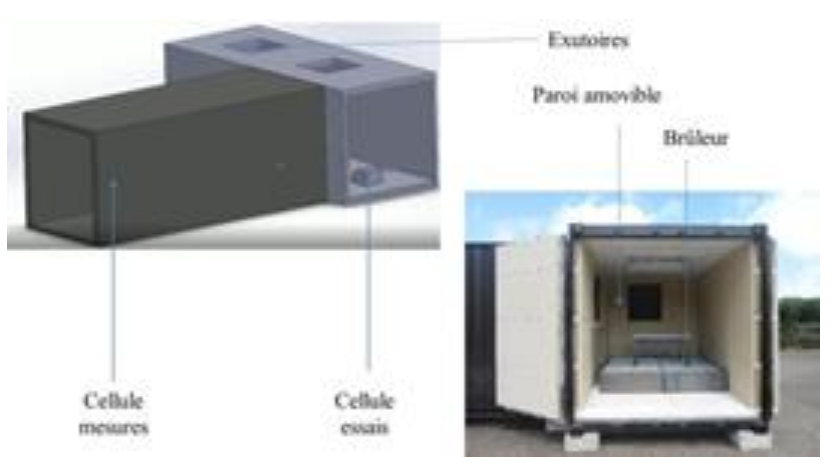

Fig. 1. Experimental cell. 
The specification of the necessary for the tender to be launched for the acquisition of dedicated cell experimental cell. These specifications take into account all the specificities related to hostile and complex constraints of flows encountered. The experimental test bench is composed of two containers. One is used for the data acquisition and the other one is for the experimental tests. We have chosen maritim containers because they have more or less the same volume we can find in a standard bedroom. The experimental container is thermally isolated. There are two windows allowing the optical acces, a mobil wall, two outlets and the fire place. These tools let study fire under different conditions: Two different volumes and ventilated or under ventilated fire. The analysis performed will consist on: Fire dynamics, Temperature fields, smoke opacity and gases concentration [9][10].
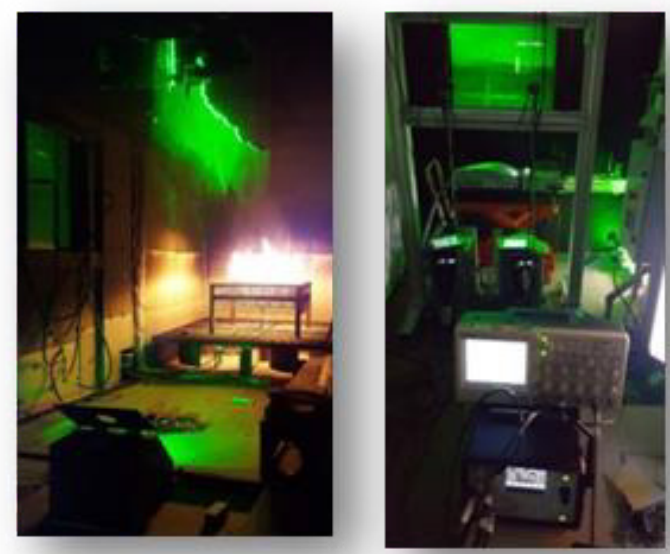

Fig. 2. Particle Image Velocimetry (PIV) Measurement Systems

\subsection{Numerical simulations}

For the numerical simulations, the calculation code Fire Dynamics Simulator (FDS) has been retained. Widely used in fire safety engineering, this code allows obtaining a faithful representation of the fire dynamics

In order to optimize the reliability of this software, meshes of $5 \mathrm{~cm}$ are used for the simulations presented later. A sketch of the numerical design is shown in Figure 3. For the experimental cell, this represents slightly more than 285,000 nodes. To optimize the computation time, the simulations are carried out using a supercomputer attached to the laboratory, CRIAAN meso calculation center.



Fig. 3. Modeling FDS of the cell

The entire set of FDS results is post processed using the Matlab software.

For this study, four main configurations are chosen different scenarios: Large volume, an open outlet; Large volume, two open outlets; Small volume, an open outlet, Small confined volume. For all the current simulations, a power of $500 \mathrm{~kW}$ is applied. For the different scenarios, the firepower can be modified. This is the case of Scenario 1 or calculations are carried out up to $1 \mathrm{MW}$. In order to compare the numerical simulations with the first experimental measurements, sensors are placed in the same places, numerically and experimentally.

\section{RESULTS}

The following section shows the development of the fire at $500 \mathrm{~kW}$ considering for Large volume, an open outlet. The fireplace is located to the right of the cell with the outlet open to the left. The conditions at the walls are considered adiabatic. For this simulation, the mesh is also made with cells of $5 \mathrm{~cm} \times 5 \mathrm{~cm}$. An instantaneous temperature field at time $\mathrm{t}=50$ after the fire starts is shown in Figure 4.

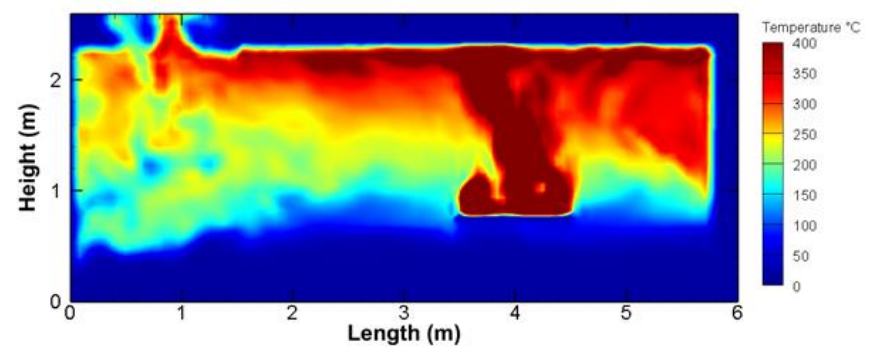

Fig 4: Modelling of the temperature at 50 seconds

At 50 seconds, the fire develops normally. There is a layer of stratified hot flue gases in the upper part of the volume concerned. At 300 seconds, Figure 5, the fire behaviour is completely different. A large part of the oxygen of the volume has been consumed. The initial focus is no longer localized at the burner Indeed; it is observed that the flame moves at the outlet position. On the modelling, the flame position is highly fluctuating. This phenomenon represents the alternation of fresh air intake and evacuation of hot smoke. 


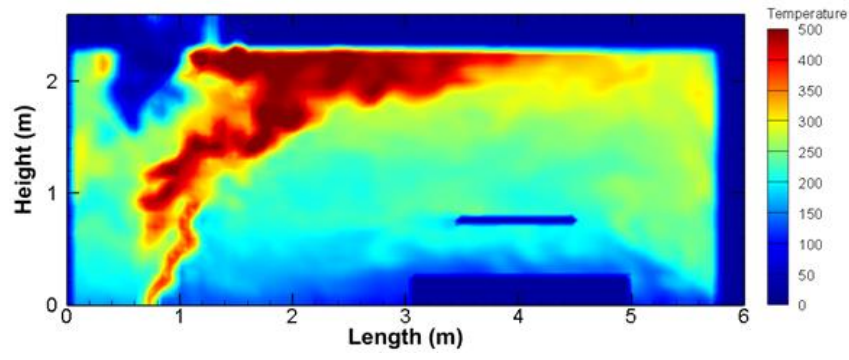

Fig. 5: Modelling of the temperature $\left({ }^{\circ} \mathrm{C}\right)$ at $300 \mathrm{~s}$

Averaging the temperature field, Figure 6, we can observe that the flame is mainly positioned at the outlet. Where there exists a. In a under ventilated fire situation, this phenomenon is explained by the lack of oxidant (here the oxygen) necessary for the home to develop properly.Indeed, when the fireplace lacks oxygen to burn the whole of the fuel present, the pressure in the enclosure decreases and will allow fresh air to enter. This fresh air inlet will restart the combustion and increase the pressure of the volume again (through the increase of smoke) which will lead to a release of hot fumes.

This phenomenon is repeated until the fuel is exhausted. It is illustrated in Figure 6 where a higher temperature is observed at the outlet as well as an absence of flame on the fireplace. However, these numerical observations remain to be verified with the experimental one. Indeed, the FDS code is limited when modeling under ventilated fires. It tends to overestimate the appearance of ghost flames (flame that comes off the burner to use the oxidizer of the room). Although this phenomenon is scientifically possible, the focus could just as well be extinguished and the flame would disappear.

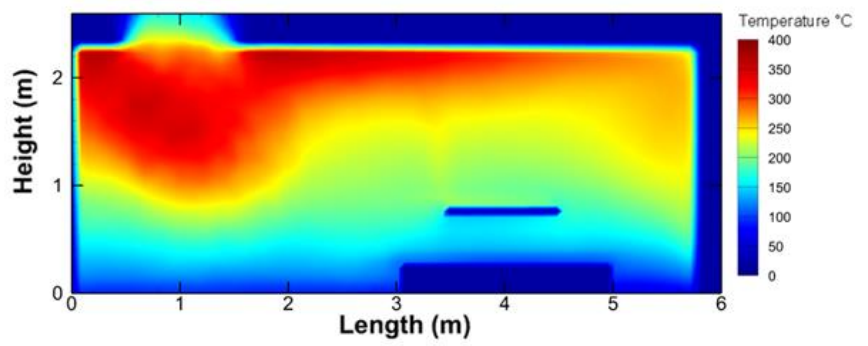

Fig. 6: Modelling of the average temperature $\left({ }^{\circ} \mathrm{C}\right)$

\subsection{Experimental test}

In order to validate the numerical results presented above, an experimental test was carried out, namely, small volume, $500 \mathrm{~kW}$, open outlet. The 36 burners were turned on. The thermocouple taken into account for the results below is placed in the same place as the digital sensor. Figure 7 shows the fireplace with the 36 burners burning. However, for better modularity, the number of burners can be changed, thus modifying the power fluxdensity.
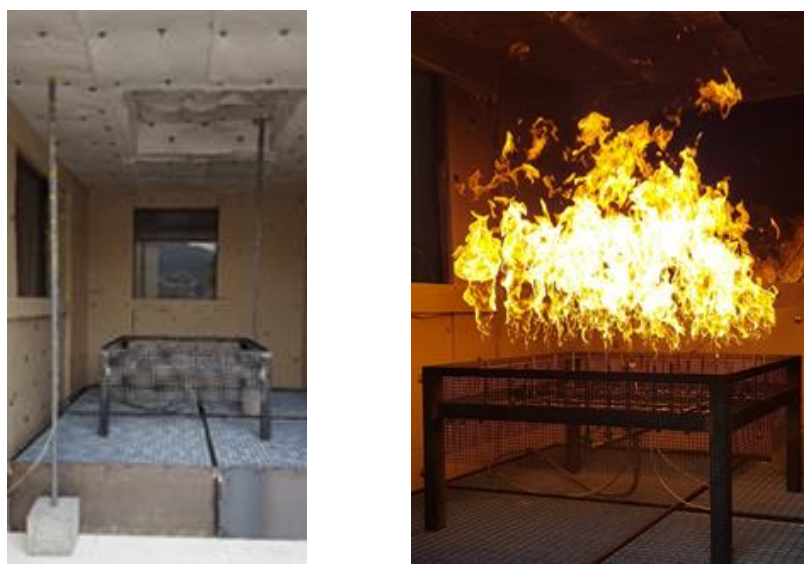

Fig 7: Test cell and Fireplace 500kW and 36 burners

Following this test, the following temperature against time curve is plotted, Figure 8 . The experimental curve is represented in red, the adiabatic in pink and the curve in the normal conditions FDS in orange.

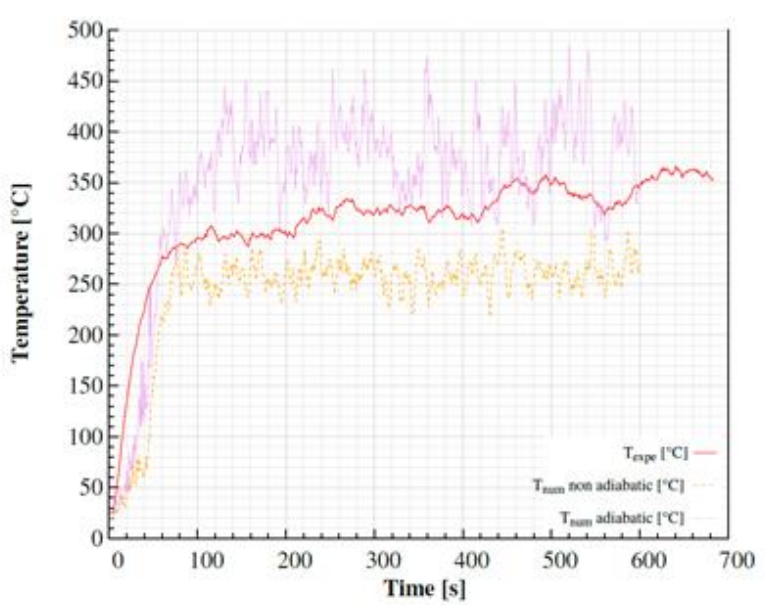

Fig.8: Curves to $500 \mathrm{~kW}$

From the simulations, adiabatic and real configurations are tested. Compared to the numerical values, Figure 8 , it can be observed that the three curves are very similar. However, the adiabatic numerical values (pink curve) are slightly higher than the experimental values, whereas the non-adiabatic case is under estimating the temperature. However, good agreement is observed. On the experimental curve, we can observe that the steady state is not reached, in 
contrast to the numerical simulations presented above. On the other hand, the rise in power is underestimated in numerical terms. In order to get closer to the experimental, improvements have to be made on numerical simulations.

\subsection{Comparison experimental and numerical}

Figure 9 shows the evolution of the temperature as a function of time. The red curve always represents the experimental value. The blue curve represents the result of the improved numerical simulation. In order to get closer to the measured temperature, three improvements were made.

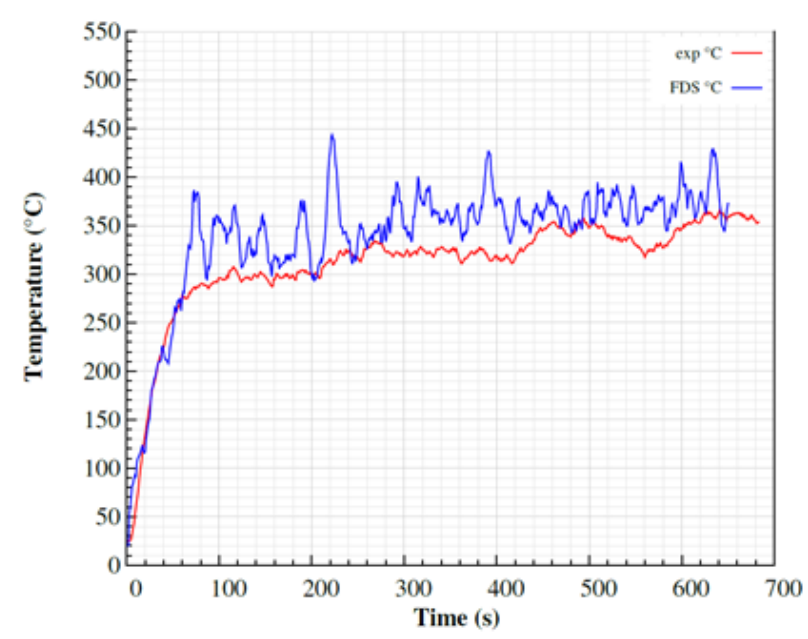

Fig. 9: Experimental and modified curves to $500 \mathrm{~kW}$

Firstly, the ramp-up has been reduced. The fuel being propane, the combustion is extremely fast. Secondly, an opening in the lower part was created. It aims to simulate the leakage observed on the cell during the tests. Thirdly, the digital firepower has been adapted. Indeed, for reasons related to the experimental installation, the power of the fireplace cannot remain constant at $500 \mathrm{~kW}$. Propane bottles are frozen and the power is changed. After checking, the firepower is closer to $460 \mathrm{~kW}$. The numerical simulation presented here was carried out with this value.Following these improvements, there is a very similar increase in power in experimental and with numerical simulation. The gap between the curves and reduces. Moreover, it can be seen that the digital curve does not reach the steady state.


Fig. 10. Comparison experimental and numerical for different power 200,500 et $800 \mathrm{~kW}$.

Comparisons of experimental and digital temperature profiles are presented in Figure 10. We note an excellent match for a power of $200 \mathrm{~kW}$, a slight offset for a power of $500 \mathrm{~kW}$ and a strong offset for a power of $800 \mathrm{~kW}$ at the first level. transient phase where the temperature gradient is very important 


\section{4 study of the Heat Release Rate (HRR)}

Figure 11 shows a study of the HRR (Heat Release Rate) was carried out to observe the numerical instabilities especially for the modeling of the under ventilated conditions. It is observed that for a fire condition underventilated (Power $800 \mathrm{~kW}$ ), the HRR oscillates around $700{ }^{\circ} \mathrm{C}$ and shows that it is difficult to use the numerical simulation under these conditions.



Fig. 11. HRR pour une puissance de 200, 500 et $800 \mathrm{~kW}$

\section{Conclusion}

To conclude, the first numerical and experimental results are conclusive. Indeed, it is observed that the temperature profiles obtained are quite similar. Technical points from the CFD need to be slightly adapted. Other measures will soon be initiated in order to compare other physical quantities and thus further refine the numerical model including speed measurements by PIV. These measurements will make it possible to compare the numerical and experimental results for the speed, another significant physical magnitude for the compartment fires. The series of experimental tests will allow to verify the numerical results obtained for configurations under ventilated and will thus validate the calculation code FDS. In addition, with the aim of checking the code, scenarios with different firepower (up to $1 \mathrm{MW}$ ) will be simulated and experimentally tested. In addition, measurements of opacimetry and a gas analyzer will complement the experimental cell. These measures will also be used to compare numerical values and thus enhance the reliability of the code.

\section{Acknowledgments}

This project has been funded with the support from the European Union with the European Regional Development Fund (ERDF) and from the Regional Council of Normandie for project FEDER MADNESS. The financial support of the Agence Nationale de la Recherche (ANR) - ANR FIREDIAG project - and the CRIANN for the simulation support are acknowledged. The fire district SDIS76 is also acknowledged for its support concerning the experimental setup.

\section{References}

[1] You, D., Jourda, P., Hattenberger, J., Vantelon, JP., Gaviot-Blanc, F., Joachim, A., Mitanchez, G. , Study of the efficiency of the fire extinguishing systems used by fire fighters on the underventilated fires with 3D Firefighting and other application techniques, Interschultz, (2010).

[2] Morton, B. R., Taylor, G. I. \& Turner, J. S. Turbulent gravitational convection from maintained and instantaneous sources. Proc. R. Soc. Lond. A234, 1 - 23, (1956).

[3] Francis, J., Chen, A.P., Observable characteristics of flashover, Fire Safety Journal, (2012).

[4] Hou, X.C., Gore, JP., Baum, HR., Measurements and prediction of air entrainment rates of pool fires, Symposium (International) on Combustion, Vol.26, Issue 1, 1453-1459, (1996).

[5] McGrattan, K., Hostikka, S., McDermott, R., Floyd, J., Weinschenk, C., Overholt, K., , Fire Dynamics Simulator Technical Reference Guide Volume 1 \& 2: Mathematical Model, NIST Special Publication, (2007).

[6] Alileche, Nassim, Damien Olivier, Lionel Estel, et Valerio Cozzani. "Analysis of domino effect in the process industry using the event tree method ». Safety Science, (2016).

[7] Juan Luis Jiménez Laredo, Frédéric Guinand, Damien Olivier, Pascal Bouvry ,: " Load Balancing at the Edge of Chaos: How Self-Organized Criticality Can Lead to Energy-Efficient Computing ". IEEE Trans. Parallel Distrib. Syst. 28(2): 517-529, (2017).

[8] L. I. Gliga, H. Chafouk,. Fault Detection and Identification for Sensors used in Confined or SemiConfined Spaces, CSCS21, 21th International Conference on Control Systems and Computer Science, 29-31 MAY 2017,Bucharest, Romania, (2017).

[9] Betting.B, Varea.E, Lebey.M, Patte-Rouland.B, Dynamique des fumées d'incendies lors de feux de compartiments : études à grande échelle et simulations numériques, GDR Feux (2017).

[10] Tieszen, S. R., O'hern, T. J., Schefer, R. W., Weckman, E. J., Blanchat, T. K., Experimental study of the flow field in and around a meter diameter methane fire, Combustion and Flame, Vol.129, Issue 4, pp. 378391, (2002). 